\title{
cDNA-library testing identifies transforming genes cooperating with c-myc in mouse pre-B cells
}

\author{
Inge Wolf, Corinne Bouquet and Fritz Melchers
}

Max Planck Fellow Research Group, “Lymphocyte Development”, Max Planck Institute for Infection Biology, Berlin, Germany

While c-myc often contributes to the generation of B cell transformation, its transgenic overexpression alone does not lead to full transformation of B-lineage cells. Synergistically acting second genes must cooperate. Here, we constructed doxycyclineinducible cDNA-libraries from pre-B cell mRNA. These libraries were retrovirally transduced as single copies into single cells and overexpressed in fetal-liver-derived c-mycoverexpressing pre-B cell lines. We scored transformation by survival and/or expansion of differentiating B-lineage cells in vitro and in vivo. Only one double c-myc/cDNAlibrary-expressing cell line was found in less than $5 \times 10^{6}$ library-transduced pre-B cells surviving and expressing a cDNA-library-derived transcript in vitro. This transcript was identified as a shortened form of the Exosc1 gene, encoding the RNA exosome complex component CSL4. Transplantations of double c-myc/Exosc1 shortform- or c-myc/Exosc1 full-length-transgenic cells into Rag1-/- mice resulted in survival, differentiation to $\mathrm{CD} 19^{+} \mathrm{CD} 3^{-} \mathrm{sIgM}^{+} \mathrm{CD} 5^{\text {low/ }}-\mathrm{CD} 11 \mathrm{~b}^{+}$mature $\mathrm{B} 1$ cells and, surprisingly, also vigorous expansion in vivo. Strikingly, after transplantations of cmyc/cDNA-library pre-BI cells the frequencies of double-transgenic pre-B cells and their differentiated progeny, expanding in vivo to heterogeneous phenotypes, was at least tenfold higher than in vitro. In a first analysis Ptprcap, Cacybp, Ndufs7, Rpl18a, and Rpl35a were identified. This suggests a strong influence of the host on B-cell transformation.

Keywords: B-lineage cell transformation · cDNA-library overexpression · c-myc $\cdot$ Exosc1

Additional supporting information may be found in the online version of this article at the publisher's web-site

\section{Introduction}

Genes that contribute to cell cycle entry, proliferation, differentiation, and apoptosis or survival of pre-BI lymphocytes and their differentiated progeny are expected to be contained in their mRNA and, hence, in cDNA-libraries generated from their mRNA. Deregulation and overexpression of some of these genes are expected to result in partial transformation on the way to malignant states,

Correspondence: Inge Wolf

e-mail: wolf@mpiib-berlin.mpg.de in which B-lineage cells show decreased apoptosis and/or proliferation, possibly at defined stages of B-cell development. By transducing pre-BI cell lines with such genes, we can follow the consecutive development towards their progeny, both in vitro and, after transplantation, in vivo.

Many B cell leukemias develop in successive steps, in which genetic and microenvironmental factors change the expression of genes regulating proliferation, differentiation and survival [1-3]. Deregulated overexpression of these oncogenes can fully transform cells at distinct stages of cellular development, and generate long-lived, expanding cells in vivo which also might be able to proliferate and/or survive as cell lines in vitro [4]. Early studies have 
already indicated, that full transformation requires at least two functionally cooperating oncogenes, one providing anti-apoptotic, the other pro-proliferative, or tumor suppressor factors-disruptive activities [5-7].

The effects of c-myc on cell cycle entry-enhancement, proliferation-activation, B-lineage cell-deregulation, and genome destabilization have been analyzed extensively [8-14]. We have previously transduced wild-type pre-BI cell lines, proliferating on stromal cells (OP9) in the presence of IL7 [15], with SIN-retroviral vectors containing doxycycline-inducible forms of cell-cycle enhancing c-myc and anti-apoptotic pim-1 [4]. When wild-type, nontransduced pre-B cell lines are removed from the stimulatory action of IL7 and OP9 stromal cells, they cease to proliferate, differentiate to pre-BII, and $\operatorname{sIgM}^{+}$immature $\mathrm{B}$ cells, and rapidly enter apoptosis [15]. Doxycycline-controlled overexpression of c-myc in pre-B cells enhanced entry into cell cycle, but did not inhibit normal apoptosis, while pim-1 overexpressing cells showed minimally reduced apoptosis, but did not proliferate. Differentiation of the nontransduced, as well as c-mycor pim-1-single transduced pre-BI cells to $\operatorname{IgM}^{+}$B cells remained normal, i.e. was induced by the removal of OP9 stroma and IL7 in vitro, or by transplantation into Rag-deficient hosts. By contrast, joint doxycycline-controlled overexpression of c-myc together with pim-1 (c-myc/pim-1) allowed differentiation to $\operatorname{IgM}^{-}$pre-BII-like cells. These cells survived and expanded by proliferation in vitro and in vivo. This expansion was reversed, when doxycycline was removed, resulting in normal development to $\operatorname{IgM}^{+}$B cells without further deregulated proliferation or survival [4].

These studies indicated that single oncogene-transgenic cells could be suitable targets to test B cell-lineage- or B-lymphomaderived cDNA-libraries for second, cooperating oncogenes. If we chose pim-1 single transduced cells, we would screen for proliferation-enhancing c-myc-like activities, while we would test for pim-1-like, survival-enhancing activities, if we used the c-myc-overexpressing cells. Here, we report our search for cDNA genes with anti-apoptotic, c-myc-complementing actions, in cmyc-transduced pre-BI cells and their progeny, induced by differentiation in vitro and in vivo.

Since the establishment of techniques for full-length cDNA cloning [16] and later full-length cDNA-library generation [17] in the 1980s, cDNA-libraries became a powerful tool for identification of new oncogenes [18-20]. Beside their identification, cDNA-library screens allow the detection of alterations within the exonic DNA, splice alterations or mutation of already known and unknown transforming genes.

We hypothesize that deregulated overexpression of many genes, might have yet unknown transforming impacts on cellular development. Therefore, we generated a cDNA-library from wildtype in vitro proliferating pre-BI cells. We chose the Gateway ${ }^{\circledR}$ cloning to transfer the cDNA-libraries to a doxycycline-inducible expression vector system (Supporting Information Fig. 1). This system allows cloning without restriction enzymes, which might corrupt some of the genes within the library [21].

We overexpressed this cDNA-library in c-myc-overexpressing pre-BI cells and screened for cells which survive in the absence of growth supporting stromal cells and IL7, i.e. undergo transformation by the cooperating actions of the cDNA-library-derived genes and c-myc.

By synergistic overexpression of c-myc and cDNA-libraryderived genes in pre-BI cells, we detected one in $5 \times 10^{6}$ cells that long-term survived without OP9/IL7. However, when these $5 \times 10^{6}$ c-myc/cDNA-library-transduced pre-BI cells were tested in vivo by transplantation into immunodeficient $\operatorname{Rag} 1^{-/-}$mice, we detected five to tenfold higher numbers of double transduced cells, indicating a major host influence in the process of transformation. These transformed cells did not only survive, but were also found to expand and to differentiate toward progressed B-lineage stages in vivo. The structural identification of this first set of in vitro and/or in vivo-transforming genes from cDNA-libraries identified a wide spectrum of proteins, which appear to destabilize their normal functions, when they are deregulated and overexpressed.

\section{Results}

\section{C-myc-transgenic pre-B cells as targets for transductions with cDNA-libraries}

The cDNA expression library was generated in three steps. First, the cDNA-library double strand fragments were reverse transcribed from mRNA of in vitro cultivated proliferating pre-BI cells [15]. The cDNA was fused to short nucleotide sequences (attB1 and attB2), which are essential for following recombination procedures into different vector systems (Supporting Information Fig. 1A). Second, the cDNA was recombined into an entry vector (Supporting Information Fig. 1B). Third, the entry library was transferred into a retroviral destination vector, forming an expression library. As destination vector a self-inactivating (SIN) retroviral vector was used (Supporting Information Fig. 1B) [4]. In this vector, gene expression is controlled by a tetracyclineresponse-element (TRE) and is induced in the presence of doxycycline (TetON) [22]. Finally, the quality of both cDNAlibraries was assessed by restriction enzyme analysis and Sanger sequencing followed by Blast and Alignment analysis (Supporting Information 2).

A previously established fetal-liver-derived pre-BI cell line by our laboratory (4) was used to screen for genes contained in our cDNA library that cooperate with c-myc in pre-BI cell transformation. This pre-BI cell line has previously been transduced with a vector encoding the reverse-transactivator (rtTA) and has been selected for high rtTA expression [4] (rtTA-pre-BI). To track doxycycline-inducible c-myc-transduced cells in vitro and in vivo, we constructed a polycistronic vector, expressing c-myc and GFP, connected by a F2A peptide (Supporting Information Fig. 3A), [24-26]. The c-myc-F2A-GFP (c-myc-GFP) plasmid was retrovirally transduced into fetal-liver derived rtTA-pre-BI cells generating a c-myc-GFP pre-BI cell line. Upon addition of doxycycline, c-myc-GFP expression was confirmed on protein levels or by fluorescence cytometry (Supporting Information Fig. 3BD). The c-myc-GFP pre-BI cell line was further transduced with 
A.

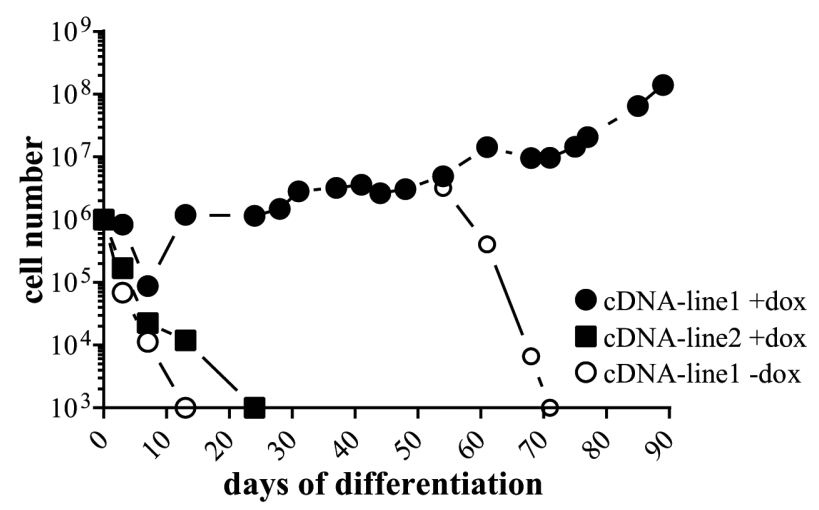

B.
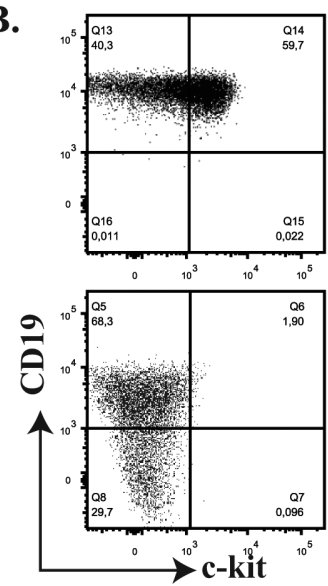

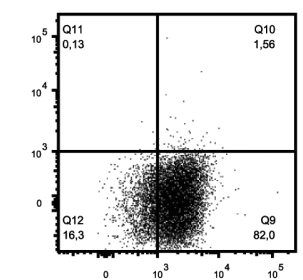

+ OP9/IL7

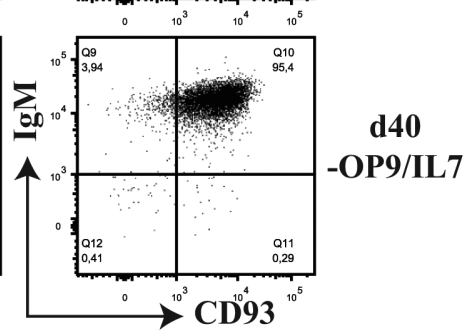

C.

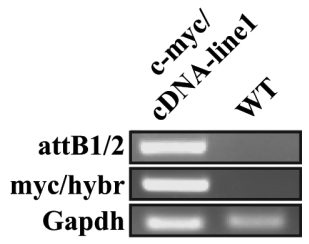

D. $\bar{x}^{80}$ Exose1

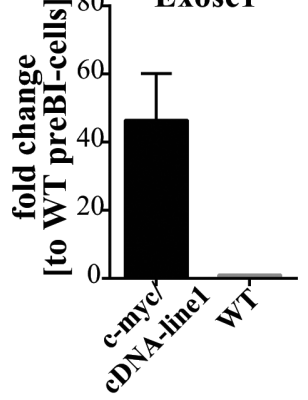

E.

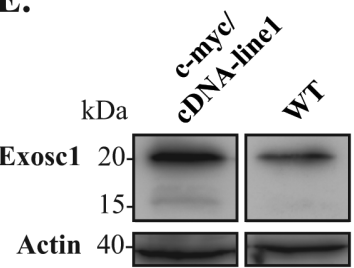

Figure 1. Identification of a shortened transcript of the Exosc1 gene cooperating with c-myc in pre-BI cell transformation in vitro. (A) Survival and differentiation analysis of c-myc and cDNA-library-line1 and 2 overexpressing pre-BI cells after OP9 and IL7 deprivation in vitro in the presence (closed circles) or absence (open circles) of doxycycline was measured by counting living cells using a hemacytometer and Trypan blue exclusion. (B) Changes in expression of CD19, c-kit, CD93 and IgM by c-myc/cDNA-library overexpressing pre-BI cells before and 40 days after OP9/IL7 deprivation were measured by flow cytometry. Gating strategy see Supporting Information Fig. 6. (C) RT-PCR with primers specifically binding attB1 and attB2 regions (attB1/2) flanking the expression cDNA-library derived gene of interest (myc/hybr, Gapdh $=$ controls). (D) qRT-PCR analyses using Exosc1 gene as internal primer. Data are shown as fold change to wild-type pre-BI cells, presented as mean \pm SEM of 5 samples. (E) Detection of Exosc1sf in cDNA-library-line1 cells by Western Blot. Actin = loading control. Data shown are representative of 3-5 (A-D) and 1 (E) independent experiment(s).

tertiary doxycycline-inducible constructs containing either no inserts (empty vector) or the cDNA library (Supporting Information Fig. 3A).

To determine the minimum cell number, necessary to detect oncogene-driven transformation, we double-transduced c-mycGFP pre-B cells with pim-1 [4] and empty vectors (Supporting Information $3 \mathrm{E} \& \mathrm{~F}$ ), mixed in different ratios. We differentiated the cells in the absence of OP9 and IL7. While c-myc and pim-1 double-transgenic pre-BI cells proliferated and differentiated to surrogate light chain expressing [23] pre-BII cells, single, nontransduced or empty vector expressing cells died [4].

For the first 28 days of differentiation, 50 c-myc/pim- 1 expressing cells were sufficient to detect oncogene-driven transformation of pre-B cells by growth. However, to detect oncogene-driven transformation beyond 28 days, $5 \times 10^{3}$ c-myc/pim- 1 doubleexpressing cells were necessary to be detectable (Supporting Information 3E).

\section{Transformation of c-myc-transgenic pre-BI cells with cDNA expression library-derived genes in vitro}

We transduced $1 \times 10^{6}$ c-myc-GFP pre-BI cells each with retroviruses containing cDNA-library-derived genes at a multiplicity of infection (MOI) of one. Subsequently, we differentiated these cells in the absence of OP9 and IL7. In five separate experiments, only one c-myc/cDNA library cell line (c-myc/cDNA-line1) survived for 20 days and beyond (Fig. 1A). Within 20 days of OP9/IL7 deprivation, the $\mathrm{GFP}^{+} \mathrm{CD} 19^{+} \mathrm{CD} 3^{+}{ }^{\mathrm{c}}$ - $\mathrm{kit}^{+} \mathrm{sIgM}^{-}$pre-BI cells had differ-

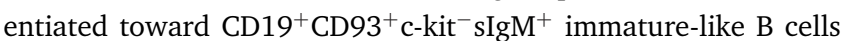
(Fig. 1B). When doxycycline was removed from culture, the cells did not survive, indicating that the transformation was dependent on overexpression of c-myc and the cDNA library-derived gene (Fig. 1A).

By RT-PCR using attB1/2 anchor primers (Fig. 1C) we amplified one single cDNA-library-derived gene product, which we 
identified by Sanger sequencing and subsequent Blast analysis as a short-form of the Exosc1 gene (Exosc1sf) (GenBank: KX371790). The Exosc1sf transcript was truncated at the C-terminal end and lacked the stop-codon (Supporting Information 4). The wild-type Exosc 1 encodes the protein exosome complex component CSL4 [29].

By quantitative real-time PCR using gene internal primers, the Exosc 1 transcript levels were 42 -fold upregulated in c-myc/cDNAline cells compared to wild-type pre-BI cells (Fig. 1D). In addition to the wild-type, full-length CSL4 protein of $21 \mathrm{kDA}$, we detected a shortened, Exosc1sf encoded, CSL4 protein of $\sim 15 \mathrm{kDa}$ in Exosc1sf-overexpressing pre-BI cells by Western Blot (Fig. 1E). The cell line c-myc/cDNA-line1 was changed to c-myc/Exosc1sf.

\section{C-myc/Exosc1sf immature-like B cells expand and differentiate to mature $\mathrm{B} 1$ cells in vivo}

Within 40 days of in vitro culture without OP9 and IL7, cmyc/Exosc1sf pre-BI cells differentiated to immature-like B cells but not beyond (Fig. 1B). To investigate, whether these cmyc/Exosc1sf cells would have the potential to differentiate towards later B-lineage stages, we transplanted $5 \times 10^{6}$ day 40 differentiated c-myc/Exosc1sf immature-like B cells into doxycyclinefed, immunodeficient (Rag1 $1^{-/}$) mice (Fig. 2A). Four weeks after transplantation, the spleen of doxycycline-fed c-myc/Exosc1sf mice increased in size (Fig. 2B). GFP ${ }^{+}$cells were detected in the bone marrow and spleen of all recipients (Fig. 2C). These data indicate that the combined overexpression of c-myc and Exosc1sf leads to an oncogenic transformation of differentiating pre-B cells in vitro, which can also be propagated in vivo. Since the transplanted cells increased in numbers, i.e. expanded, it suggested that the host provided stimulating influence and increased efficiency of B-cell expansion.

We further tested, whether mutagenic insertions of retroviral vectors into c-myc-GFP-transduced pre-BI cells, could account for the development of transformed cells. Hence, pre-BI cells, doubletransduced with c-myc-GFP and an empty vector (c-myc/empty vector) (Supporting Information Fig. 3A\&B), were generated, and their expansion in vivo after transplantation was monitored (Fig. 2C). One hundred-fold lower numbers of $\mathrm{GFP}^{+}$cells were detected, predominately as pre-BI cells, in bone marrow and spleen, when compared with the numbers of $\mathrm{GFP}^{+}$cells, detected in mice transplanted with immature-like B cells transduced with c-myc and Exosc1sf. We conclude that, at most $1 \%$ of all $\mathrm{GFP}^{+}$ cells might have developed from transplanted cells with mutagenic insertions of the retroviral vector, and that $99 \%$ of these $\mathrm{GFP}^{+}$cells developed as a result of the dual overexpression of c-myc and cDNA-library-derived Exosc1sf. This also confirmed previous results [4] showing that overexpression of c-myc alone is insufficient to induce transformation, ligand-independent survival or proliferation of B-lineage cells in vitro and in vivo.

The transferred c-myc/Exosc1sf immature B cells were predominately detected as $\mathrm{CD}_{1}{ }^{+} \mathrm{c}-\mathrm{kit}^{-} \mathrm{sIgM}^{+} \mathrm{CD} 93^{+}$ immature B cells and as $\mathrm{CD}_{19}{ }^{+} \mathrm{ckit}^{-} \mathrm{CD}^{-}{ }^{-} \mathrm{sIgM}^{+} \mathrm{IgD}^{-} \mathrm{CD} 21^{-}$
$\mathrm{CD}^{-} 3^{-} \mathrm{CD}^{\text {low/- }} \mathrm{CD}^{-11 b^{+}}$B-lineage cells (Fig. 2C). As we would expect [30], these data indicate that these fetal-liver-derived cells differentiated toward mature B1 cells in vivo. Furthermore, slightly increased IgM but no IgG concentrations were detected in sera of doxycycline-fed mice 4 weeks after transplantation of cmyc/Exosc1sf cells (Fig. 2D). Doxycycline-fed mice transplanted with c-myc/Exosc1sf immature-like B cells died on average 60 days after transplantation (Fig. 2E). Control mice, transplanted with c-myc/Exosc1sf-transduced immature-like B cells in the absence of doxycycline, did not expand $\mathrm{GFP}^{+}$cells in the bone marrow or spleen, nor did their sera contain elevated levels of IgM or IgG. These data indicate that the expansion of $\mathrm{GFP}^{+}$ $\mathrm{B}$-lineage cells in vivo and the elevated levels of IgM are a result of c-myc/Exosc1sf co-expression pre-BI cells and their differentiated progeny.

When we isolated $\mathrm{GFP}^{+}$c-myc/Exosc1sf transplanted cells from bone marrow and spleen of doxycycline-fed mice and cultured them in vitro in the absence or presence of doxycycline, the cells did not expand and survived at least for 14 days (Fig. 2F). This indicates that the transplanted host contributed essential expansion- and differentiation-stimulating influences on the c-myc/Exosc1sf cells.

\section{Transforming activity of the full-length Exosc1 transcript}

To test, whether the full-length Exosc1 could also have transforming activity, we transduced c-myc-GFP pre-BI cells with a retroviral doxycycline-inducible plasmid encoding full-length Exosc1 (Exosc1fl) cDNA (Supporting Information Fig. 5). Simultaneous overexpression of c-myc and Exosc1fl, again, induced antiapoptotic activity in vitro after OP9/IL7 deprivation, though less strongly than the truncated form (Fig. 3A).

Four weeks after transplantation of c-myc/Exosc1fl pre-BI cells into doxycycline-fed Rag $1^{-/-}$recipients, $\mathrm{GFP}^{+}$cells accumulated in the bone marrow and spleen (Fig. 3B), however in tenfold lower numbers compared to transplantations with c-myc/Exosc1sf transformed immature B cells (Fig. 2C). Most $\mathrm{GFP}^{+}$cells gave rise to a heterologous population of $\mathrm{CD}_{19}{ }^{+} \mathrm{c}-\mathrm{kit}^{+} \mathrm{CD} 93^{+} \mathrm{sIgM}^{-}$ pre-BI cells, CD19 ${ }^{+}$c-kit ${ }^{-} \mathrm{CD} 3^{+} \mathrm{sIgM}^{+}$immature-like B cells and $\mathrm{CD} 9^{+}$ckit $^{-} \mathrm{CD}^{-} 3^{-} \mathrm{sIgM}^{+} \mathrm{CD} 21^{-} \mathrm{CD}_{23}{ }^{-} \mathrm{CD}^{\text {low }}-\mathrm{CD} 11 \mathrm{~b}^{+}$mature B1 cells (Fig. 3B). Reduced IgM concentrations and no IgG were detected in serum of doxycycline-fed mice transplanted with c-myc/Exosc1fl pre-B cells (Fig. 3C).

We conclude that the full length Exosc1 gene can cooperate with c-myc to transform B-lineage cells, though less efficiently than the short-form Exosc1.

\section{Pre-BI cell pools co-overexpressing c-myc and cDNA-library genes expand in vivo to different stages}

In previous experiments we found, that the in vivo environment of transplanted Rag1 ${ }^{-/-}$recipients exerts proliferative influence 

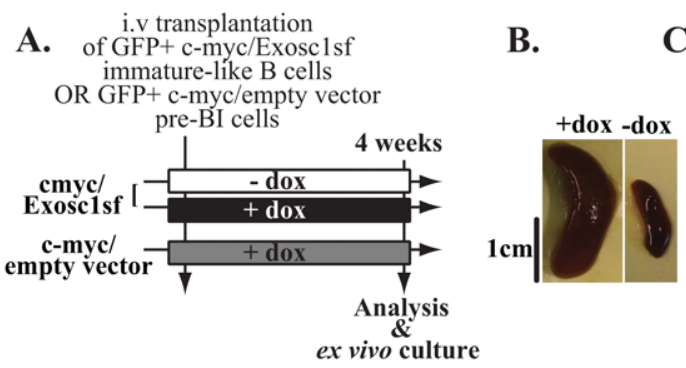

D.

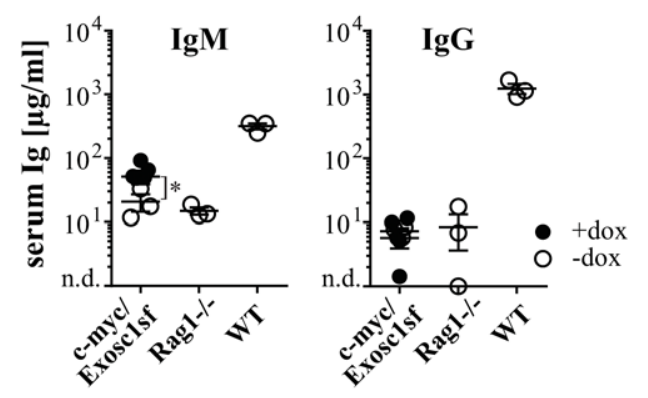

E.

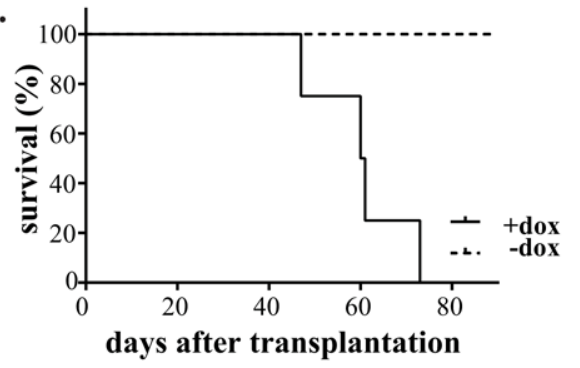

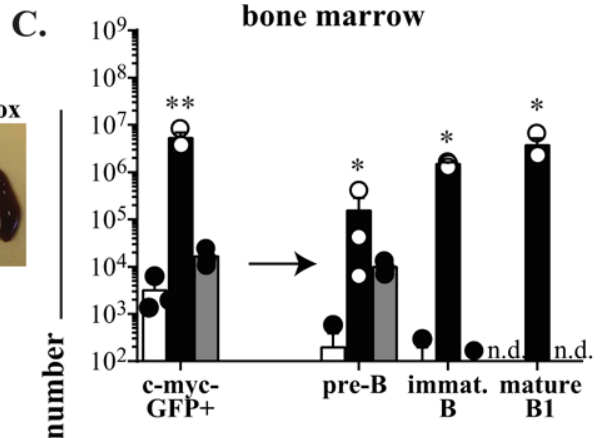

$\overline{\bar{z}}$

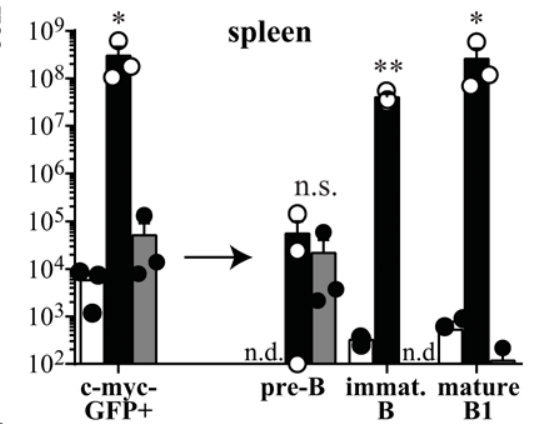

F.

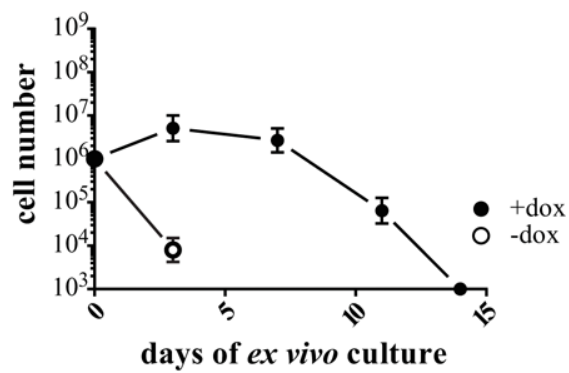

Figure 2. C-myc/Exosc1sf immature-like B cells expand and differentiate to mature B1 cells in vivo. (A) Transplantation schedule of GFP ${ }^{+} \mathrm{c}^{-}$ myc/Exosc1sf immature-like B cells into doxycycline-fed (black bars) and non-fed (open bars) immunodeficient (Rag1 ${ }^{-l-}$ ) mice. As control, one group of doxycycline-fed Rag1 $1^{-/}$mice was transplanted with $\mathrm{GFP}^{+}$pre-BI cell lines overexpressing c-myc and an empty, doxycyclineinducible vector (c-myc/empty vector). (B) Splenomegaly detected in doxycycline-fed immunodeficient recipients (+dox, closed circles) compared to the nondoxycycline-fed control group (-dox, open circles). (C) FACS analysis of bone marrow (1 hind leg) and spleen-derived GFP ${ }^{+}$ c-myc transgenic B-lineage cells 4 weeks after transplantation of c-myc /Exosc1fl pre-BI cells into immunodeficient Rag1 ${ }^{-/-}$mice. The c-myc$\mathrm{GFP}^{+}$cells were subgated (arrow pointing right) on $\mathrm{CD} 19^{+}{ }^{+} \mathrm{ckit}^{+} \mathrm{CD} 93^{+}$pre-BI cells, $\mathrm{CD} 19^{+} \mathrm{ckit}^{-} \mathrm{CD} 3^{+} \mathrm{sIgM}^{+} \mathrm{CD} 21^{-} \mathrm{CD} 23^{-}$immature B cells, and $\mathrm{CD}_{19}{ }^{+} \mathrm{ckit}^{-} \mathrm{CD} 93^{-} \mathrm{sIgM}^{+} \mathrm{CD} 21^{-} \mathrm{CD} 23^{-} \mathrm{CD} 5^{\text {low/-}} \mathrm{CD} 11 \mathrm{~b}^{+}$mature B1 cells. Dots indicate cell numbers detected in individual mice. n.d. $=$viable cells not detected. (D) ELISA of IgM and total IgG in sera of transplanted mice 4 weeks after transplantation. Rag1 ${ }^{-1-}$ and wild-type (WT) C57BL/6 $=$ controls, n.d. = Ig secretion not detected. (E) Survival of doxycycline-fed c-myc/Exosc1sf transplanted mice (solid line; $n=10$ ) compared to their control group which received no doxycycline (dashed line; $n=5$ ) (Kaplan-Maier analysis). (F) Ex vivo differentiation culture of bone marrow derived GFP ${ }^{+}$cells in the presence (closed circles) or absence (open circles) of doxycycline. (A-F) Data are shown as mean \pm SEM of $n=3$ and 5 mice/group/experiment and are representative of two independent experiments. Statistical significance was determined by 1-way ANOVA (C) and two-tailed unpaired t-test (D); ${ }^{*} p<0.05,{ }^{* *} p<0.005$.

on the in vitro nonexpanding c-myc/Exosc1sf and c-myc/Exosc1fl cells. Therefore, we next screened c-myc/cDNA-library doubletransduced pre-B cells for additional cDNA-library-derived genes, which might be susceptible to host stimulation for in vivo expansion after transplantation.

In a second round of c-myc-GFP pre-BI cell-transfection, new doxycycline-inducible c-myc/cDNA-library double-transgenic preBI-cell lines were generated. Again, $5 \times 10^{6}$ of the c-myc/cDNAlibrary gene-transduced pre-BI cells were induced to differentiate in vitro upon OP9/IL7 deprivation, while another $5 \times 10^{6}$ of these cells were directly transplanted into Rag $1^{-/-}$recipients (Fig. 4A), both in the presence of doxycycline. As seen before, simultaneous overexpression of c-myc and cDNA-library genes had a weak antiapoptotic, and no proliferation-inducing effects on the collection of double-transgenic pre-BI cells (Fig. 4B). The cells did not survive beyond day 20 after removal of OP9/IL7.

Surprisingly, and in contrast to transplantations of c-myc/Exosc1sf and c-myc/Exosc1fl-transgenic B-lineage cells, not only pre-BI, immature and matureB1 cells were detected. The mice transplanted with c-myc/cDNA-library genes-transduced pre$\mathrm{BI}$ cells were found to contain additionally, heterogeneous pools of cells resembling $\mathrm{CD}_{19}{ }^{+/ \text {low }} \mathrm{CD} 138^{+} \mathrm{IgM}^{+} \mathrm{TACI}^{-} \mathrm{MHC}^{\text {high} /+}$ plasmablasts and $\mathrm{CD} 19^{\text {low/- }} \mathrm{CD} 138^{+} \mathrm{IgM}^{-} \mathrm{TACI}^{+} \mathrm{MHC}^{\text {low/- }}$ plasma cells (Fig. 4C). 
A.

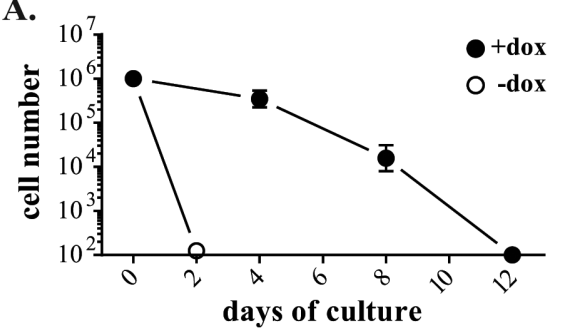

C.

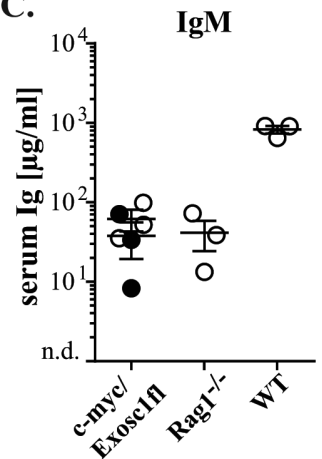

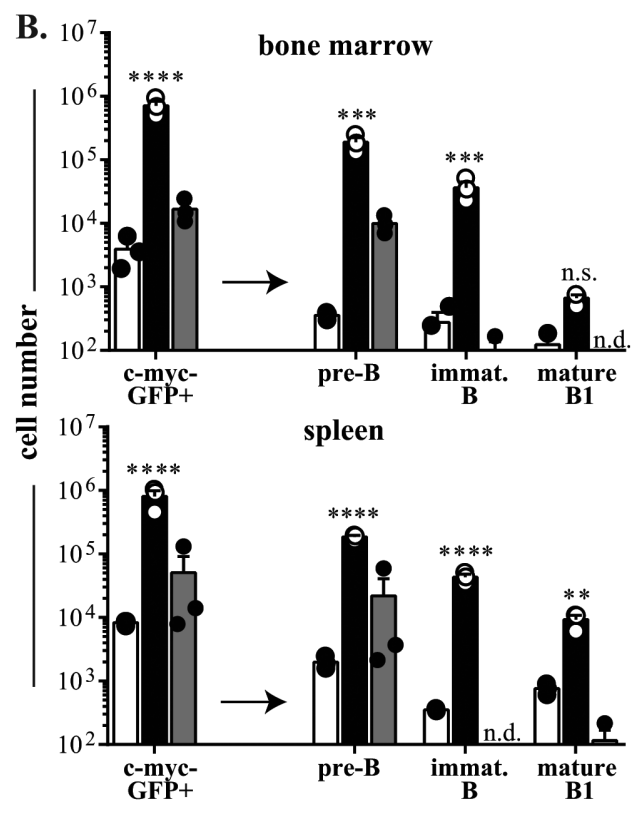

Figure 3. C-myc/Exosc1fl pre-BI cells expand and differentiate to mature B cells in vivo. (A) Differentiation of c-myc/Exosc1fl overexpressing pre-BI cells upon OP9/IL7 deprivation in the presence (closed circles) or absence (open circles) of doxycycline in vitro, enumerated by counting living cells using a hemacytometer and Trypan blue exclusion. (B) FACS analysis of bone marrow (1 hind leg) and spleen derived GFP ${ }^{+}$B-lineage cells 4 weeks after transplantation of c-myc/Exosc1fl (black bars) or c-myc/empty vector (grey bars) pre-BI cells into doxycycline-fed (closed symbols) or non-fed (open symbols) immunodeficient Rag1-1- mice. The c-myc-GFP ${ }^{+}$cells were sub-gated (arrow pointing right) on $\mathrm{CD}^{-19} \mathrm{ckit}^{+} \mathrm{CD} 93^{+}$

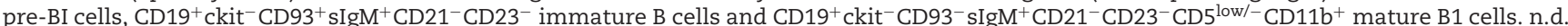
$=$ viable cells not detected. (C) ELISA of IgM and total IgG in doxycycline-fed transplanted mice 4 weeks after. Rag1 ${ }^{-1-}$ and wild-type (WT) C57BL/6 $=$ controls, n.d. $=$ Ig secretion not detected. Data are shown as mean \pm SEM of $n=3$ mice/group/experiment and are representative of two independent experiments. Statistical significance was determined by 1 -way ANOVA; ${ }^{* *} p<0.005,{ }^{* * * *} p<0.0005,{ }^{* * * *} p<0.0001$, n.s. $=$ statistically not significant.

Spleen-derived $\mathrm{GFP}^{+}$cells of some, but not all of these mice expressed increased amounts Blimp1, a master regulator of terminal B-cell development to plasma cell differentiation [31], while other mice harbored $\mathrm{GFP}^{+}$cells expressing AID transcripts, which is restricted to germinal center B cells [32] (Fig. 4D). By contrast, Blimp1 and AID transcripts were not found in spleens from doxycycline-fed mice transplanted with c-myc/Exosc1sf or c-myc/Exosc1fl cells. This suggests, that the c-myc/Exosc1 transformed cells were all arrested in vivo as $\operatorname{sIgM}^{+}$mature B1 cells and did not differentiate toward later B-lineage stages, while some of the cells transfected with cDNA genes of the library developed plasmablasts and plasma cells.

Furthermore, in some, although not all mice reconstituted with c-myc/cDNA-library cells, elevated levels of IgM, but not IgG, were detected in the sera (Fig. 4E). The heterogeneous gene expression patterns and Ig serum levels coincide with the heterogeneous FACS phenotypes of transplanted $\mathrm{GFP}^{+}$c-myc/cDNA-library cells.

After transplantations of c-myc/cDNA-library-overexpressing pre-BI cells, heterogeneous pools of differentiated B-lineage cells developed in vivo. These heterogeneous pools of doubletransduced cells differentiated either to immature B and mature B1 cell stages, or proceeded to later, germinal center-like or plasmablast/plasma-like cell stages. Our data indicate that this B-lineage heterogeneity might be a result of different sets of cDNA-library-derived genes that cooperate with c-myc in cell transformations. Single cell cloning of the double-transduced Blineage cells ex vivo, followed by re-transplantation, will have to be done to resolve this heterogeneity of transformed cells, and to determine, which cDNA-library-genes induce differentiation to mature B cells, and which other genes allow further differentiation to plasmablasts and plasma cells.

\section{Identification of cDNA-library genes cooperating with c-myc in B-cell transformation in vivo}

Next, $\mathrm{GFP}^{+}$cells from bone marrow were isolated ex vivo and analyzed for abundance of cDNA-library-derived genes by RTPCR. Several different transcripts of different sizes were amplified from the RNA of $\mathrm{GFP}^{+}$bone marrow cells from each of the transplanted mice (Fig. 5). This indicates that the pool of transplanted, in vivo expanded c-myc/cDNA-library cells expressed several different cDNA-library-derived genes in each of the transplanted recipients. Five cDNA-library-derived PCR products were sequenced (Fig. 5). They were identified as Rpl18a, Rpl35a Ndufs7, Cacybp, and Ptprcap. All sequences were full-length and without mutations.

Based on the results described above, we conclude that the frequency of genes which support c-myc in B cell transformation in vivo is much higher than in vitro, thus suggesting that the 
A.

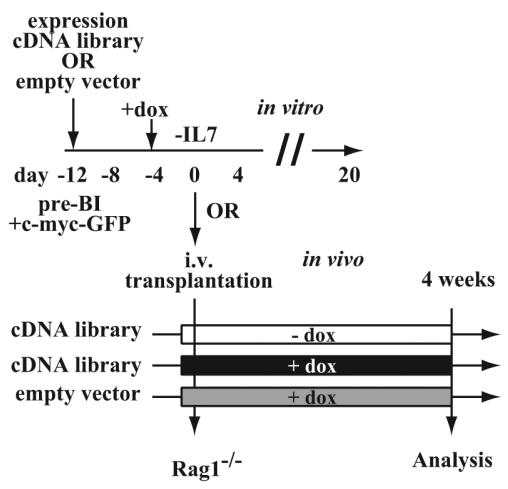

C.

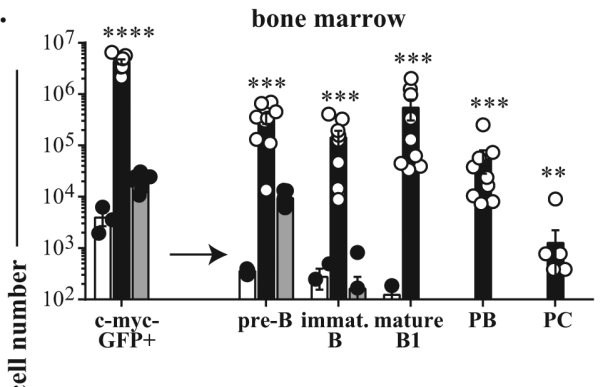

B.

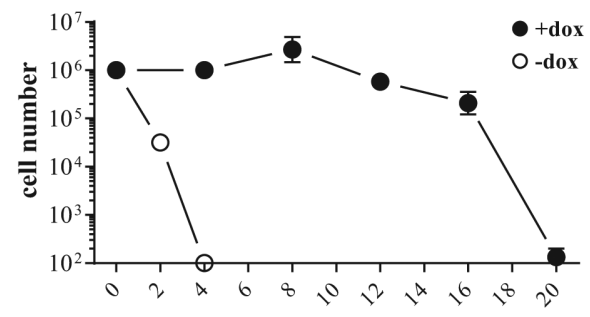

days of in vitro differentiation

D.
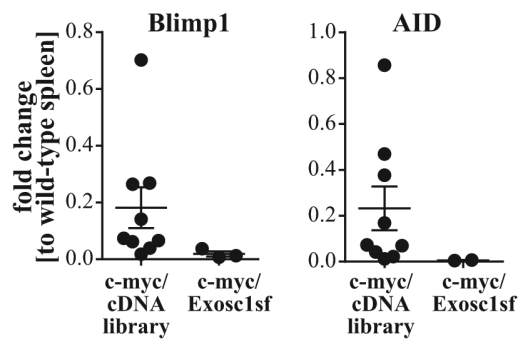

E.
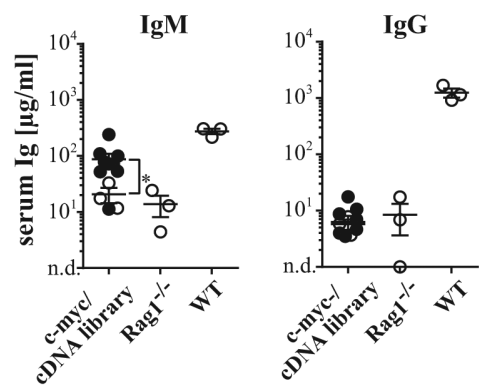

Figure 4. Transplantation of c-myc/cDNA-library overexpressing pre-BI cells. (A) In vitro culture and transplantation schedule of c-myc/cDNAlibrary transgenic pre-BI cells. (B) Differentiation analysis of c-myc/cDNA-library overexpressing pre-BI cells upon OP9/IL7 deprivation in the presence (closed circles) or absence (open circles) of doxycycline in vitro. (C) FACS analysis of bone marrow (1 hind leg) and spleen derived GFP ${ }^{+}$ c-myc/cDNA-library cells 4 weeks after transplantation into doxycycline-fed (black bars) or doxycycline-non-fed (open bars) Rag1-/- mice. As control, one group of doxycycline-fed Rag1 ${ }^{-/-}$mice was transplanted with GFP ${ }^{+}$pre-BI cell lines overexpressing c-myc and an empty, doxycyclineinducible vector (c-myc/empty vector, gray bars). The c-myc-GFP ${ }^{+}$cells were subsequently gated for pre-B cells $\left(\mathrm{CD} 19^{+} \mathrm{C}-\mathrm{kit}^{+} \mathrm{CD} 93^{+} \mathrm{IgM}{ }^{-}\right)$, immature

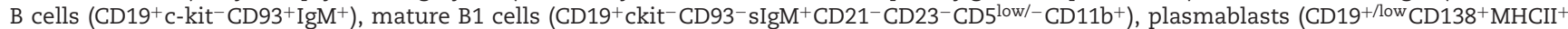
$\mathrm{IgM}^{+}$) and plasma cells (CD19low/- $\mathrm{CD} 138^{\mathrm{hi}} \mathrm{MHCII}^{-} \mathrm{IgM}^{-} \mathrm{TACI}^{+}$). Gating strategy see Supporting Information Fig. 7. (D) qRT-PCR of GFP ${ }^{+}$cell-derived RNA from spleens of c-myc/cDNA-library pre-BI cells or c-myc/Exosc1sf immature-like B cell chimeras. Data are presented as fold change of mRNA levels relative to wild-type spleen cells (E) ELISA of IgM and total IgG in sera of transplanted mice 4 weeks after transplantation of c-myc/cDNAlibrary overexpressing pre-BI cells. Rag1 ${ }^{-1-}$ and wild-type (WT) C57BL/6 = controls, n.d. $=$ Ig secretion not detected. (B-E) Data are shown as mean \pm SEM of $n=6$ and 9 and 3 (control) mice/experiment and are representative of two independent experiments. Statistical significance was determined by 1 -way ANOVA (C) and two-tailed unpaired t-test $(\mathrm{E}){ }^{* *} p<0.005,{ }^{* * *} p<0.0005,{ }^{* * * *} p<0.0001$.

host provides a transformation-stimulating environment for the development of malignant B cells

\section{Discussion}

An extended analysis of one cDNA-library-derived gene with transforming activity in vitro and in vivo has identified a shortened transcript variant of the Exosc1 gene [33-35].

The wild-type form of Exosc1 is ubiquitously expressed [27, 28] and encodes the protein exosome complex component 1 (CSL4), a core component of the RNA exosome complex which is essential for 3' mediated mRNA degradation, processing and quality control [29].

Since the short-form was not found to be a product of alternative splicing, and did not contain a translation termination codon it poses the question, how such an RNA could be translated into a short protein form, that we detected in Western Blots (Fig. 2). When a message without a stop codon is freed from its association with ribosomes, the growing polypeptide chain behind it could also be freed in this "nonstop decay" reaction. Future experiments should determine, which of the "nonstop decay" mechanisms [36] could operate in c-myc/Exosc1sf-overexpressing pre-BI cells and their progeny to allow the expression of a truncated, stop codon 


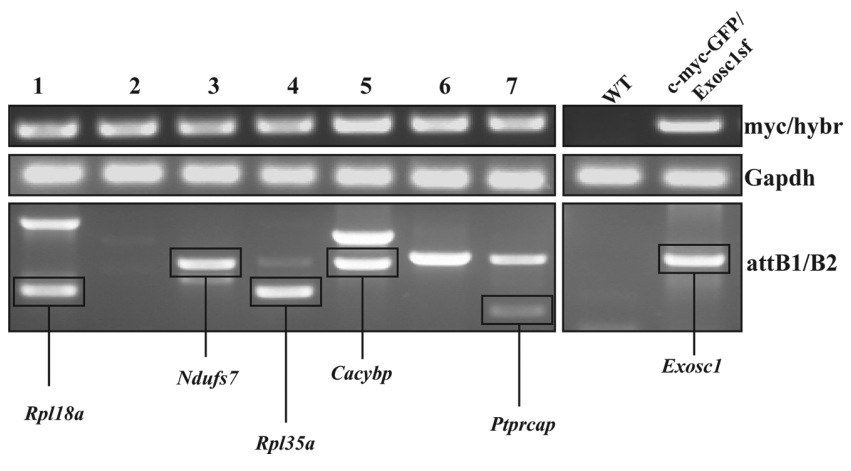

Figure 5. Identification of cDNA-library-derived genes. RT-PCR of GFP ${ }^{+}$ cells derived from bone marrow of c-myc/cDNA-library transplanted mice. Products were amplified using attB1/B2 cDNA-library specific primers. Numbers indicate one individual mouse, $\mathrm{WT}=$ wild-type bone marrow (negative control), c-myc/Exsc1sf = positive control. Gapdh = loading control. Data shown represent two independent experiments performed with 9 and 6 mice, respectively.

missing CSL4 protein, and how the truncated protein, or the fulllength protein, disturbs the functions of the exosome.

The RNA exosome consists of a catalytically inactive, barrelshaped core composed of nine subunits (six subunits form a $\mathrm{PH}$-ring, which is assembled only in the presence of three cap proteins) and one additional unit harboring catalytic activity [37]. The RNA exosome complex component CSL4, is one of the three cap proteins and contains three domains: N-terminal RPL27 providing contact with the PH-ring, a central S1-domain binding mRNA substrate and essential cofactors, and a C-terminal zincribbon-like domain essential for mRNA decay [33, 38, 39]. The S1-domain and the zinc-ribbon-like domain have been found to be dispensable for exosome assembly. Thus, the zinc-ribbon-domaindefective CSL4 protein, produced by Exosc1sf, could still assemble in the exosome, bind RNA, but be unable to degrade mRNA.

In B-lineage cells, overexpression of this shortened CSL4 protein would compete with the endogenously expressed wild-type CSL4 for the binding to the PH-ring and in fact, might replace it. Hence, mRNAs could not be degraded at normal rates, but their concentrations in the transduced cells would increase, leading to enhanced synthesis of proteins. The increased concentration of those proteins, which stimulate survival and/or proliferation of B-lineage cells, would affect increased longevity and expansion.

We have also seen, that the wild-type full-length Exosc1 transcript encoding a full-length CSL4 protein, with an intact zincribbon-like domain, transforms B-lineage cells in vitro and in vivo, indicating that even the full-length CSL4 could interfere with proper mRNA degradation, when overexpressed in B-lineage cells. Under these circumstances, high levels of CSL4 proteins might interfere with the proper formation of the RNA exosome complex, thereby reducing RNA decay activity. Again, this would lead to increased mRNA concentrations in the cell, and result in enhanced translation of, among others, anti-apoptotic and proliferationstimulating proteins. In addition, high concentrations of free CSL4 proteins might bind mRNAs, inhibiting their proper delivery to the exosome and, thus, their degradation, again with the result of, increased translation.
Our results offer fascinating possibilities to study functions of the exosome complex in situations, where individual components of the complex are defective and/or overexpressed.

Transplantations of c-myc/Exosc1sf, c-myc/Exosc1fl and cmyc/cDNA-library genes indicate that the B-lineage cells not only survive, but also expand by proliferation in vivo. It suggests that the host provides growth- and differentiation-promoting influences. It has long been known that leukemic B-lineage cells, which expand vigorously in vivo and which, in the case of mouse plasmacytomas [40], can be easily transplanted [41], resist proliferation in vitro and rapidly die in cultures. Hence, the host contributes essential stimulation to B-cell leukemias for in vivo proliferation of these malignant cells. The same differences have been observed with human B-cell leukemias. Tissue cultures containing selective cytokines, feeder layer and additional nutritional requirements, which are essential for the establishment and cloning of human malignant lymphoma cell lines, have improved conditions to establish plasmacytoma and lymphoma cell-lines [42, 43]. Overexpression of Exosc1sf, which we show to convey long-term survival to mouse B-lineage cells, in human B-cell leukemias, might further be of help to establish in vitro cell lines from such primary ex vivo tumor cells.

Our in vivo screenings have further identified five cDNAlibrary-derived genes (Rpl18a, Rpl35a Ndufs7, Cacybp, and Ptprcap) that potentially transform B-lineage cells, in cooperation with c-myc. One of the five identified cDNA genes, Cycybp, in fact is a c-myc target gene [44]. C-myc is predicted to target and regulate around $11-15 \%$ genes of the genome $[45,46]$. It is possible, that the c-myc target genes could be preferred cooperating oncogenes in the c-myc-transduced pre-BI cells. Therefore, the identification of a much higher number of cDNA genes would be necessary. However, we do not think that our method for finding cooperating oncogenes selects c-myc targets, since we overexpress nontransformed, i.e. "healthy", in vitro proliferating pre-BI cells. Second transforming, malignancy-propagating genes in in vivo development of B-lineage malignancies might well be selected not only for their function to be transforming (by different types of mutations), but also be selected by deregulated c-myc expression acting via myc-target sequences for myc-sensitivity of their expression. This is not the case in our experiments. Hence, our transformation protocols are likely not selecting for such mycsensitivity, as an in vivo development of B-lineage tumors might do.

Our results show that single oncogene (e.g. c-myc-) transduced pre-B cells can be used to screen cDNA-libraries for proto-oncogene-mediated cooperating second activity that, when overexpressed, promote development, survival and expansion of B-lineage cells. Their activities are reminiscent of pleiotropically acting mutations in epigenetic modifiers and anti-apoptotic genes that are introduced early in common precursors of follicular lymphomas [47]. Many oncogenes have been previously characterized in B-lymphomas and multiple myelomas with survival- or proliferation-promoting activities of B-lineage cells. Surprisingly, our screen detects a multitude of genes, which were, so far, not considered to be proto-oncogenes. This opens large fields of 
further investigations into normal and deregulated actions of many functions in a B-lineage cell.

\section{Materials and methods}

\section{Cell culture}

RtTA-transduced pre-BI cells, OP9 stroma cells and Plat-E packaging cell line (Life Technologies) were cultivated as previously described [4, 48]. Differentiation of pre-BI cells was induced upon removal of OP9 stroma cells and IL7, or of IL7 alone.

\section{Transduction of pre-BI cells}

Retroviral vectors were transiently transfected into Plat-E cells using Lipofectamine (Life Technologies) as previously described [4]. The gene expression was achieved by addition of $1.5 \mu \mathrm{g} / \mathrm{mL}$ doxycycline into the culture medium.

\section{Vectors}

Doxycycline-inducible, "TreTight"-TetOn-controlled [49] SIN retroviral vectors encoding c-myc-, pim-1-, the cDNA-library, Exosc1fl or no insert (empty vector) were constructed as reported [4] (Supporting Information Fig. 1B and Fig. 3A). The c-myc used in our studies is a mouse-human hybrid sequence that allows differential detection of endogenous and transduced c-myc in RT-PCR analyses. For simultaneous expression of c-myc and GFP (c-myc-GFP), a polycistronic vector was constructed, which expressed both genes from one transcript, connected by sequences encoding the 22 amino acid-long 2A peptide (Supporting Information 3, A), a cis-acting hydrolase element that allows skipping between the conserved glycine and proline [24-26]. After processing, the expression of this vector yields a c-myc protein with one additional amino acid from the $2 \mathrm{~A}$ peptide at the amino-terminal end, and a longer than normal GFP protein with 20 amino acids from the $2 \mathrm{~A}$ peptide at the carboxy-terminal end.

For the generation of a doxycycline-inducible expression vector the attR flanked ccdB gene and Chloramphenicol resistance gene $\left(\mathrm{Cm}^{\mathrm{R}}\right)$ was amplified from destination vector (pDEST14, Life Technologies) and inserted into the linearized vector. pENTR $^{\mathrm{TM}}$ 1A (Life Technologies) was used for the generation of the entry library. Sequence of inserted genes was verified by Sanger sequencing (Eurofins MWG Operon Sequence).

\section{Western blot}

Western Blot analysis were performed as previously described [4]. Exosc1 was detected using anti-mouse Exosc1 (EPR13525, Abcam).

\section{RNA and mRNA purification}

Total RNA was purified using TRIzol ${ }^{\circledR}$ Reagent (Life Technologies). For cDNA-library generation RNA of $2 \times 10^{8}$ fetal-liver derived pre-BI cells cultivated on OP9 stroma and IL7 was purified followed by mRNA isolation using the FastTrack ${ }^{\circledR}$ MAG mRNA Isolation Kit (both from Life Technologies) according to the manufacturer's instructions. For RT- and qPCR RNA of $5 \times 10^{5}-1 \times 10^{7}$ cells was extracted. The mRNA concentration and purity was estimated by spectrophotometry (NanoDrop 1000, Peqlab).

\section{Generation of a cDNA library}

The full-length cDNA-library was generated according to the SuperScript ${ }^{\circledR}$ Full Length cDNA-library Construction Kit II (Life Technologies) protocol. If not noted otherwise, all reagents were supplied with the kit. Between 5 and $10 \mu \mathrm{g}$ mRNA were used the production of a cDNA-library containing $1.0 \times 10^{7}$ to $1.2 \times 10^{7}$ primary cDNA clones in E.coli. The plasmid DNA from bacterial colonies was purified using Plasmid Miniprep Kit (Sigma, Qiagen, Hilden; Germany) and the heterogeneity was confirmed by BsrGI (entry library) or XhoI (expression library) (New England Biolabs, Ipswich MA, USA) digestion. Sequence analysis were performed with following primers: Entry library 5'-TGTAAAACGACGGCCAGT-3' and 5'-CAGGAAACAGCTATGACC-3',

Expression library 5'-TGATAGAGAACGTATGTCGAGGTAG-3' and $5^{\prime}$-TATCCAGCCCTCACTCCTTCTC-3'. The sequence results were blasted in Nucleotide BLAST followed by sequence alignment with the matching homologue mRNA sequence and coding DNA sequence (CDS) derived from Nucleotide database (ncbi.nlm.nih.gov/nuccore) using SeqMan Pro software (DNAStar ${ }^{\circledR}$, Madison, WI, USA). Functional annotation analysis was performed using DAVID functional annotation tool (https://david.ncifcrf.gov). For detailed cDNA-library qualification analyses see Supporting Information 2.

\section{Semiquantitative and quantitative real-time RT-PCR}

For cDNA preparation, equal amounts of RNA and dilutions thereof were used for each condition. Transcription of RNA into cDNA was performed using SuperScript III reverse transcriptase (Life technologies) and oligodT or random hexamer primers (Thermo Fisher Scientific, Waltham, MA, USA). Amplification of cDNA was performed using primers specific for:

myc/hybr 5'-CGGATTCTCTGCTCTCCTCGAC-3' and 5'-CGT CGAGGAGAGCAGAGAATCC-3', Gapdh 5'-TTGAGGTCAATGAAG GGGTC-3' and 5'-TCGTCCCGTAGACAAAATGG-3', attB1/B2 5'-GCCTGGAGAATTCGGATCTAGAT-3' and $5^{\prime}$-ACC ACTTTGTACAAGAAAGTTGGGT-3'

pInsert $5^{\prime}$-TCCTCCCTTTATCCAGCCCT-3' and $5^{\prime}$-TGTACGG TGGGAGGCCTATA-3'

Exosc1 5'-GGAGCTGTCGTCACCTGTAA-3' and 5'-GCTCGGA TATCTTCTTTGCGG-3' 
IgG1 5'-GACCTCTACACTCTGAGCAGC-3' and 5'-GGGGAAGA TGAAGACAGATGAT-3'

IgM 5'-ACCTGAATGTGTACACCTGCC- $3^{\prime}$ and $5^{\prime}$-AGGAAGA TGTCGGCAAAGGAG-3'

AID $5^{\prime}$-TAGTGCCACCTCCTGCTCAC-3' and $5^{\prime}$-AGGAGGTGA ACCAGGTGACG-3

Blimp1 5'-TTGGTACACACAGGAGAGAAGCC-3' and $5^{\prime}$ TAGTGTTTCTGCAGGTGGGC-3 $3^{\prime}$

RT-PCR was performed using Taq DNA Polymerase (Thermo Fisher Scientific), while quantitative RT-PCR was conducted with QuantiTect SYBR Green PCR Kit (QIAGEN) according to manufacturer's protocol.

\section{Flow cytometry}

Cells were stained with anti-mouse CD19-BV650 (6D5), B220BV510 (RA3-6B2), CD93-PerCPC5.5 (AA4.1), CD138-BV421 (281-2), c-kit-AlexaFlour700 (ACK4), IgK-PE (RMK-45), IgG1-bio (RMG1-1), IgG2a-bio (RMG2a-62), IgG2b-bio(RMG2b-1) (BioLegend, San Diego CA, USA) and CD25-PeCy7 (eBio3C7), IgMAPC or -PeCy7 (II/41), TACI-APC (ebio8F10-3) (eBioscience, San Diego CA, USA) in the presence of anti-FCgRII (2.4G2, inhouse). Streptavidin-Qdot605 (Molecular Probes) was used to visualize biotin-conjugated primary antibodies. Lymphocates were analyzed using an LSRII FACS (BD Biosciences) in the presence of DAPI (Carl Roth GmbH). Aggregates and doublets were gated out [4]. Acquisition was performed using the DiVa software 6.1 (BD Biosciences). Analysis was performed using the FlowJo software (Tree Star, Ashland OR, USA).

\section{Transplantation of pre-BI and differentiated cells into Rag1 $^{-/-}$recipients}

All experiments were performed as already described [4]. All animal procedures were conducted in compliance with the German animal protection laws with the protocol approved by the Landesamt fur Gesundheit und Soziales, Berlin (G0140-11).

\section{ELISA}

Immunoglobulin (Ig) levels of serum samples, compared with standard Ig class samples, were determined with Maxisorp ELISA 96-well plates coated with goat anti-mouse IgM or total IgG as prescribed by Southern Biotech, Birmingham AL, USA. Using APlabeled goat anti-mouse IgM-AP, or total IgG-AP antibody (Southern Biotec) the serum Igs were quantified.

Acknowledgements: We thank Simon Fillatreau (DRFZ, Berlin) and Marko Knoll (Whitehead Institute, Cambridge MA) for critical reading of our manuscript.
This work was supported by a Kosellek grant of the DFG (ME 2764-1/1) to F.M.

Conflict of interest: The authors declare no financial or commercial conflict of interest.

\section{References}

1 Scott, D. W. and Gascoyne, R. D., The tumour microenvironment in B cell lymphomas. Nat. Rev. Cancer 2014. 14: 517-534.

2 Rosean, T. R., Tompkins, V. S. Olivier, A. K., Sompallae, R., Norian, L. A., Morse, H. C., Waldschmidt, T. J. and Janz, S., The tumor microenvironment is the main source of IL-6 for plasma cell tumor development in mice. Leukemia 2015. 29: 233-237.

3 Tai, Y. T., Acharya, C., An, G., Moschetta, M., Zhong, M. Y., Feng, X. and Cea, M., APRIL and BCMA promote human multiple myeloma growth, chemoresistance, and immunosuppression in the bone marrow microenvironment. Blood 2016. 127: 3225-3236.

4 Bouquet, C. and Melchers, F., Pim1 and Myc reversibly transform murine precursor B lymphocytes but not mature B lymphocytes. Eur J Immunol. 2012. 42: 522-532.

5 Land, H., Parada, L. F. and Weinberg, R. A., Tumorigenic conversion of primary embryo fibroblasts requires at least 2 cooperating oncogenes. Nature 1983. 304: 596-602

6 Aukema, S. M., Siebert, R., Schuuring, E., van Imhoff, G. W., KluinNelemans, H. C., Boerma, E. J. and Kluin, P. M., Double-hit B cell lymphomas. Blood 2011. 117: 2319-2331.

7 Eischen, C. M., Weber, J. D., Roussel, M. F., Sherr, C. J. and Cleveland, J. L., Disruption of the ARF-Mdm2-p53 tumor suppressor pathway in Mycinduced lymphomagenesis. Genes Dev. 1999. 13: 2658-2669.

8 Dalla-Favera, R., Bregni, M., Erikson, J., Patterson, D., Gallo, R. C. and Croce, C. M., Human c-myc onc gene is located on the region of chromosome 8 that is translocated in Burkitt lymphoma cells. Proc Natl Acad Sci U S A 1982. 79: 7824-7827

9 Taub, R., Kirsch, I., Morton, C., Lenoir, G., Swan, D., Tronick, S., Aaronson, S. and Leder, P., Translocation of the C-Myc Gene into the Immunoglobulin Heavy-Chain Locus in Human Burkitt-Lymphoma and Murine Plasmacytoma Cells. Proceedings of the National Academy of Sciences of the United States of America-Biological Sciences, 1982. 79: 7837-7841.

10 Bouchard, C., Staller, P. and Eilers, M., Control of cell proliferation by Myc. Trends Cell Biol. 1998. 8: 202-206.

11 Langdon, W. Y., Harris, A. W., Cory, S. and Adams, J. M., The c-myc oncogene perturbs B lymphocyte development in E-mu-myc transgenic mice. Cell 1986. 47: 11-18

12 Park, S. S., Kim, J. S., Tessarollo, L., Owens, J. D., Peng, L., Han, S. S. and Tae Chung, S., Insertion of C-Myc into Igh induces B cell and plasma-cell neoplasms in mice. Cancer Res. 2005. 65: 1306-1315.

13 Sander, S., Calado, D. P., Srinivasan, L., Kochert, K., Zhang, B. C., Rosolowski, M. and Rodig, S. J., Synergy between PI3K signaling and MYC in Burkitt lymphomagenesis. Cancer Cell 2012. 22: 167-179.

14 Ott, G., Rosenwald, A. and Campo, E., Understanding MYC-driven aggres sive B cell lymphomas: pathogenesis and classification. Blood 2013. 122 3884-3891.

15 Rolink, A., Kudo, A., Karasuyama, H., Kikuchi, Y. and Melchers, F., Longterm proliferating early pre B cell lines and clones with the potential to develop to surface Ig-positive, mitogen reactive B cells in vitro and in vivo. EMBO J. 1991. 10: 327-336. 
16 Okayama, H. and Berg, P., High-efficiency cloning of full-length Cdna. Mol. Cell. Biol. 1982. 2: 161-170.

17 Gubler, U. and Hoffman, B. J., A simple and very efficient method for generating Cdna libraries. Gene 1983. 25: 263-269.

18 Soda, M., Choi, Y. L., Enomoto, M., Takada, S., Yamashita, Y., Ishikawa, S. and Fujiwara, S. I., Identification of the transforming EML4-ALK fusion gene in non-small-cell lung cancer. Nature 2007. 448: 561-566.

19 Ko, J., Lee, Y. H., Hwang, S. Y., Lee, Y. S., Shin, S. M., Hwang, J. H. and Kim, J., Identification and differential expression of novel human cervical cancer oncogene HCCR-2 in human cancers and its involvement in p53 stabilization. Oncogene 2003. 22: 4679-4689.

20 Shindoh, N., Yoda, A., Yoda, Y., Sullivan, T. J., Weigert, O., Lane, A. A. and Kopp, N., Next-generation cDNA screening for oncogene and resistance phenotypes. Plos One 2012. 7: e49201.

21 Landy, A., Dynamic, structural, and regulatory aspects of lambda sitespecific recombination. Annu. Rev. Biochem. 1989. 58: 913-949.

22 Urlinger, S., Baron, U., Thellmann, M., Hasan, M. T., Bujard, H. and Hillen, W., Exploring the sequence space for tetracycline-dependent transcriptional activators: novel mutations yield expanded range and sensitivity. Proc. Natl Acad. Sci. USA 2000. 97: 7963-7968.

23 Kudo, A. and Melchers, F., A 2nd gene, Vpreb in the Lambda-5 locus of the mouse, which appears to be selectively expressed in lymphocytes-Pre-B. ЕМВО J. 1987. 6: 2267-2272.

24 Delogu, A., Schebesta, A., Sun, Q., Aschenbrenner, K., Perlot, T. and Busslinger, M., Gene repression by Pax5 in B cells is essential for blood cell homeostasis and is reversed in plasma cells. Immunity 2006. 24: 269281.

25 Laemmli, U. K., Cleavage of structural proteins during the assembly of the head of bacteriophage T4. Nature 1970. 227: 680-685.

26 de Felipe, P., Polycistronic viral vectors. Curr. Gene Ther. 2002. 2: 355-378.

27 Wang, M., Weiss, M., Simonovic, M., Haertinger, G., Schrimpf, S. P., Hengartner, M. O. and von Mering, C., PaxDb, a database of protein abundance averages across all three domains of life. Mol. Cell Proteomics 2012. 11: 492-500.

28 Heng, T. S., Painter, M. W. and C.Immunological Genome Project, The Immunological Genome Project: networks of gene expression in immune cells. Nat. Immunol. 2008. 9: 1091-1094.

29 Raijmakers, R., Noordman, Y. E., van Venrooij, W. J. and Pruijn, G. J. M., Protein-protein interactions of hCsl4p with other human exosome subunits. J. Mol. Biol. 2002. 315: 809-818.

30 Hayakawa, K., Hardy, R. R., Herzenberg, L. A. and Herzenberg, L. A., Progenitors for Ly-1 B cells are distinct from progenitors for other B cells. J. Exp. Med. 1985. 161: 1554-1568.

31 Shaffer, A. L., Lin, K. I., Kuo, T. C., Yu, X., Hurt, E. M., Rosenwald, A. and Giltnane, J. M., Blimp-1 orchestrates plasma cell differentiation by extinguishing the mature B cell gene expression program. Immunity 2002. 17: 51-62.

32 Greeve, J., Philipsen, A., Krause, K., Klapper, W., Heidom, K., Castle, B. E. and Janda, J., Expression of activation-induced cytidine deaminase in human B cell non-Hodgkin lymphomas. Blood 2003. 101: 3574-3580.

33 Schaeffer, D., Tsanova, B., Barbas, A., Reis, F. P., Dastidar, E. G., SanchezRotunno, M., Arraiano, C. M. and van Hoof, A., The exosome contains domains with specific endoribonuclease, exoribonuclease and cytoplasmic mRNA decay activities. FEBS J. 2009. 276: 218-219.

34 Houseley, J., LaCava, J. and Tollervey, D., RNA-quality control by the exosome. Nat. Rev. Mol. Cell Biol. 2006. 7: 529-539.
35 Chen, C. Y., Gherzi, R., Ong, S. E., Chan, E. K. L., Raijmakers, R., Pruijn, G. J. M. and Stoecklin, G., AU binding proteins recruit the exosome to degrade ARE-containing mRNAs. Cell 2001. 107: 451-464.

36 Klauer, A. A. and van Hoof, A., Degradation of mRNAs that Lack A Stop Codon: A Decade Of Nonstop Progress. Wiley Interdisciplinary Reviews-RNA, 2012. 3: 649-660.

37 Liu, Q. S., Greimann, J. C. and Lima, C. D., Reconstitution, activities, and structure of the eukaryotic RNA exosome. Cell 2006. 127: 12231237.

38 van Hoof, A. et al., Function of the ski4p (Csl4p) and Ski7p proteins in 3'-to-5' degradation of mRNA. Mol Cell Biol. 2000. 20: 8230-8243.

39 van Hoof, A., Frischmeyer, P. A., Dietz, H. C. and Parker, R., Exosomemediated recognition and degradation of mRNAs lacking a termination codon. Science 2002. 295: 2262-2264.

40 Potter, M., Neoplastic development in plasma cells. Immunol Rev. 2003. 194: 177-195.

41 Potter, M., Experimental plasmacytomagenesis in mice. HematologyOncology Clinics of North America 1997. 11: 323-347.

42 Horibata, K. and Harris, A. W., Mouse myelomas and lymphomas in culture. Exp. Cell Res. 1970. 60: 61-77.

43 Nilsson, K., Georgii-Hemming, P., Spets, H. and Jernberg-Wiklund, H., The control of proliferation, survival and apoptosis in human multiple myeloma cells in vitro. Mechanisms of B Cell Neoplasia 1998 1999. 246: 325-333.

$44 \mathrm{Kim}$, J., Lee, J. H. and Iyer, V. R., Global identification of Myc target genes reveals its direct role in mitochondrial biogenesis and its E-box usage in vivo. PLoS One 2008. 3: e1798.

45 Fernandez, P. C., Frank, S. R., Wang, L., Schroeder, M., Liu, S., Greene, J., Cocito, A. and Amati, B., Genomic targets of the human c-Myc protein. Genes Dev. 2003. 17: 1115-29.

46 Li, Z. R., Van Calcar, S., Qu, C. X., Cavenee, W. K., Zhang, M. Q. and Ren, B., A global transcriptional regulatory role for c-Myc in Burkitt's lymphoma cells. Proceedings of the National Academy of Sciences of the United States of America, 2003. 100: 8164-8169.

47 Pasqualucci, L., Khiabanian, H., Fangazio, M., Vasishtha, M., Messina, M., Holmes, A. B. and Ouillette, P., Genetics of follicular lymphoma transformation. Cell Rep. 2014. 6: 130-40.

48 Simmons, S., Knoll, M., Drewell, C., Wolf, I., Mollenkopf, H. J., Bouquet, C. and Melchers, F., Biphenotypic B-lymphoid/myeloid cells expressing low levels of Pax5: potential targets of BAL development. Blood 2012. 120: 3688-3698.

49 Schonig, K., Bujard, H. and Gossen, M., The power of reversibility regulating gene activities via tetracycline-controlled transcription. Methods Enzymol, 2010. 477: 429-453.

Abbreviations: dox: Doxycycline - fl: full-length · rtTA: reverse transactivator · sf: short form - TRE: Tetracycline response element

Full correspondence: Inge Wolf, Max-Planck-Institut fur Infektionsbiologie, Lymphocye Development, Charitéplatz 1 Berlin, Germany 10117

e-mail: wolf@mpiib-berlin.mpg.de

Received: 21/3/2016

Revised: $28 / 6 / 2016$

Accepted: 16/8/2016

Accepted article online: 19/8/2016 